\title{
Prospective 2-year study of accelerated pulsed transepithelial corneal crosslinking outcomes for Keratoconus
}

\author{
Mohammed Ziaei ${ }^{1} \cdot$ Hans Vellara' $^{1}$ Akilesh Gokul ${ }^{1}$ - Dipika Patel ${ }^{1} \cdot$ Charles N. J. McGhee $^{1}$
}

Received: 28 October 2018 / Revised: 27 January 2019 / Accepted: 24 April 2019 / Published online: 4 July 2019

(c) The Author(s), under exclusive licence to The Royal College of Ophthalmologists 2019

\begin{abstract}
Aims To report 2-year outcomes of transepithelial, accelerated, pulsed, corneal crosslinking (t-ACXL) for patients with progressive keratoconus.

Methods Prospective, interventional case series at a university hospital tertiary referral centre. Forty eyes with progressive keratoconus undergoing t-ACXL were included. Treatment was performed with pulsed illumination ( $1 \mathrm{~s} \mathrm{on} / 1 \mathrm{~s}$ off) using 45 $\mathrm{mW} / \mathrm{cm}^{2}$ for $5 \mathrm{~min}$ and $20 \mathrm{~s}$, for a surface dose of $7.2 \mathrm{~J} \mathrm{~cm}^{2}$. Uncorrected distance visual acuity (UDVA), corrected distance visual acuity (CDVA), manifest refraction spherical equivalent (MRSE), corneal tomography, anterior segment optical coherence tomography (OCT) and confocal microscopy were evaluated preoperatively and at 12 and 24 months postoperatively.

Results The mean patient age was $23.32 \pm 5.18$ years (SD) (range 14-42 years). The mean CDVA significantly improved from $0.38 \pm 0.32 \log$ MAR at baseline to $0.30 \pm 0.21 \log$ MAR at 24 months $(P<0.01)$. There was no significant difference in UDVA, MRSE, asymmetry indices, tomographic parameters and endothelial density. The improvement in visual acuity was inversely correlated with preoperative CDVA and preoperative $\mathrm{K}_{\mathrm{Max}}$. No complications were encountered.

Conclusions In this prospective study, t-ACXL appeared safe and effective in halting progression of keratoconus within a follow-up period of 24 months.
\end{abstract}

\section{Introduction}

Keratoconus is a progressive, non-inflammatory corneal disorder that leads to myopia, irregular astigmatism and reduced visual acuity $[1,2]$. Corneal crosslinking (CXL) has rapidly become the treatment of choice to arrest the progression of corneal ectasia since it was first reported in 2003 [3].

CXL has a complex mechanism of action but in brief, it increases the stiffness of the cornea through a photochemical reaction between riboflavin and ultraviolet A (UVA) in the corneal stroma, which results in the formation of new covalent bonds between collagen fibril amino-acids $[4,5]$. Animal studies have shown a $70 \%$ increase in corneal

Mohammed Ziaei

M.ziaei@auckland.ac.nz

1 Department of Ophthalmology, New Zealand National Eye Centre, Faculty of Medical and Health Sciences, University of Auckland, Private Bag 92019, Auckland 1142, New Zealand rigidity following treatment, and similar human studies have demonstrated a $328 \%$ increase in stiffness $[3,6]$.

The standard CXL protocol, typically referred to as the "Dresden protocol", was initially reported by Wollensak et al. and requires epithelial removal, application of riboflavin $0.1 \%$ solution for $30 \mathrm{~min}$ before UV-A exposure, with a wavelength of $370 \mathrm{~nm}$ and homogenous irradiance of $3 \mathrm{~mW} / \mathrm{cm}^{2}$ for $30 \mathrm{~min}\left(5.4 \mathrm{~J} / \mathrm{cm}^{2}\right)$ [3]. More recent CXL techniques have departed from the original Dresden protocol. These newer techniques use different formulations and delivery methods of riboflavin to avoid epithelial debridement (transepithelial CXL), as well as high-intensity UV-A exposure (accelerated CXL); to shorten duration times, reduce patient discomfort, and minimise postoperative complications such as infection, stromal haze and endothelial toxicity [7].

Accelerated CXL (ACXL) reduces treatment time by taking advantage of the Bunsen-Roscoe law of photochemical reciprocity, which states that the same photochemical effect can be achieved with a reduced irradiation interval provided the total energy level is kept constant through a corresponding increase in irradiation intensity [7]. 
Experimental data has revealed that the law of photochemical reciprocity is only valid for illumination intensities not exceeding $50 \mathrm{~mW} / \mathrm{cm}^{2}$ with treatments not lasting longer than 2 min [8]. However, continuous ACXL has been shown to result in lower clinical and experimental efficacy compared to conventional CXL, and this has been attributed to the higher consumption and subsequent shortage of oxygen, and subsequent depletion of reactive oxygen species and oxidation protein products which are key drivers of the crosslinking process in the cornea [9-14]. This led to the development of a pulsed ACXL protocol in which pulsed delivery of the UV-A light is hypothesised to allow more oxygen to diffuse into the corneal stroma and lead to an enhanced release of reactive oxygen species, and thus facilitate a more effective crosslinking process [15].

Previous studies have reported favourable outcomes with both accelerated and transepithelial CXL procedures [16-21], but to the best of the authors' knowledge, there is no study in the literature reporting long-term outcomes of transepithelial, accelerated, pulsed CXL (t-ACXL) for the treatment of keratoconus. In the present study, we report the 2-year outcomes of t-ACXL for the treatment of patients with documented progressive keratoconus.

\section{Methods}

This prospective study enrolled subjects with keratoconus undergoing t-ACXL from August 2014 to October 2015 attending the University of Auckland Cornea and External Eye Disease Service, Greenlane Hospital, Auckland District Health Board, Auckland, New Zealand.

Inclusion criteria included patients with progressive keratoconus that was diagnosed based on clinical and associated tomographic findings. Progressive keratoconus was defined as one or more of the following changes: an increase of $1.00 \mathrm{D}$ or more in the steepest keratometry $\left(\mathrm{K}_{\mathrm{Max})}\right.$ measurement, an increase of $1.00 \mathrm{D}$ or more in manifest cylinder, or an increase of $0.50 \mathrm{D}$ or more in manifest refraction spherical equivalent (MRSE) over 12 months. The grade of keratoconus was staged according to the Topographic Keratoconus Classification (TKC) system which classifies keratoconus into 5 grades: 0 (normal) to 4 (severe). Exclusion criteria included contraindications to CXL including corneal thickness of $<375 \mu \mathrm{m}$ at the thinnest point, corneal scarring or oedema visible on slitlamp examination, severe dry eye, prior ocular surgery, trauma, pregnancy or lactation, systemic autoimmune disease and diabetes mellitus. Contact lens wearers were required to remove their contact lens at least 2 weeks before clinical examinations and CXL procedure.

Study examinations were undertaken at baseline, days 3-5 and months 1, 3, 6, 12 and 24 postoperatively. Preoperative and postoperative year 1 and year 2 measurements were used in the analyses of the current study.

The study was approved by the local Health and Disability Ethics Committee, a branch of the Ministry of Health in New Zealand (NTX/08/08/070AM02). Written, informed consent was obtained from all patients after they voiced understanding of the purpose and the procedures of the study in accordance with the Declaration of Helsinki.

\section{Patient assessment}

Both eyes of all patients were examined preoperatively and at 1 month, 3 months, 6 months, 12 months and 24 months postoperatively. At each visit, uncorrected distance visual acuity (UDVA) and corrected distance visual acuity (CDVA) were recorded using a logarithm of the minimum angle of resolution (LogMAR) Snellen chart. Patients were also evaluated using slit-lamp biomicroscopy, corneal tomography (Pentacam, Oculus, Germany), in vivo confocal microscopy (Confoscan 4; NIDEK Technology, Italy) and Spectralis anterior segment optical coherence tomography (OCT) imaging (Heidelberg Engineering $\mathrm{GmbH}$, Germany).

\section{Surgical technique}

The surgical procedure was conducted under sterile conditions. Two drops of topical proxymetacaine hydrochloride 0.5\% (Alcon Laboratories Inc, Fort Worth, TX) were administered and a speculum was placed between the eyelids. The corneal epithelium was left intact and Paracel solution (Avedro, Waltham, MA, USA) containing $0.25 \%$ riboflavin-5-phosphate, hydroxypropyl methylcellulose, sodium edetate, trometamol, benzalkonium chloride was applied to the corneal surface every 90 seconds for $4 \mathrm{~min}$. The cornea was then rinsed completely with VibeX Xtra (Avedro) containing $0.25 \%$ riboflavin-5-phosphate and $\mathrm{NaCl}$, and the cornea soaked with VibeX Xtra every 90 seconds for $6 \mathrm{~min}$.

Crosslinking was conducted using the Avedro UV-A source (Avedro Inc., Waltham, MS, USA) with $5 \mathrm{~min}$ and $20 \mathrm{~s}$ of pulsed illumination (1 s on/1 s off) using $45 \mathrm{~mW} / \mathrm{cm}^{2}$ for a total surface dose of $7.2 \mathrm{~J} \mathrm{~cm}^{2}$. Treated eyes were medicated with ciprofloxacin $0.3 \%$ (Alcon Laboratories Inc, Fort Worth, TX) and fluorometholone $0.1 \%$ (Santen Pharmaceutical Co, Ltd) eye drops four times a day for 1 week and 1 month, respectively. No contact lens was used.

\section{Statistical analysis}

Statistical analysis was performed using SPSS 19.0 for Windows (SPSS, IBM, Chicago, IL, USA). A sample size calculation was performed to distinguish a difference of at 
Table 1 Demographics of patients included in the study

\begin{tabular}{ll}
\hline Parameters & Value \\
\hline Patients $(n)$ & 40 \\
Age $(\mathrm{y})$ & \\
$\quad$ Mean \pm SD & $23.32 \pm 5.18$ \\
Range & $14-42$ \\
Eye & \\
Right & $40 \%$ \\
Left & $60 \%$ \\
Keratoconus stage & \\
$1-2$ & $10 \%$ \\
$2-3$ & $40 \%$ \\
$3-4$ & $50 \%$ \\
Contact lens use & \\
No contact lens & $45 \%$ \\
RGP & $32.5 \%$ \\
Semi-scleral & $15 \%$ \\
Soft & $7.5 \%$ \\
\hline
\end{tabular}

least 1.0 D in $\mathrm{K}_{\mathrm{Max}}$ between follow-up time points within the same group at a significance level of 0.05 , a power of $80 \%$ and assumption of a standard deviation of $1.85 \mathrm{D}$. The assumption of the standard deviation was determined from the mean standard deviation of $\mathrm{K}_{\mathrm{Max}}$ from the repeatability of $\mathrm{K}_{\mathrm{Max}}$ using the Pentacam HR in eyes with keratoconus $[22,23]$. This produced a minimum sample size of 29 . The distributions of the variables were evaluated by the Kolmogorov-Smirnov test. Paired $t$-test was used for comparisons. Pearson correlation coefficients were used to analyse the possible correlation between preoperative visual and tomographic parameters and outcomes. A $P$ value $<$ 0.05 was considered significant.

\section{Results}

Forty eyes of 40 patients with keratoconus were included in the study. No significant intraoperative/postoperative complications or adverse reactions were observed. Temporary foreign body sensation following CXL was commonly reported in the first 1-3 days after treatment. No patient developed an epithelial defect or clinically significant corneal haze.

Preoperative characteristics of the patients in each treatment group are demonstrated in Table 1 . Two patients $(5 \%)$ were older than 35 years of age at the time of treatment. The mean changes in UDVA and CDVA after t-ACXL are depicted in Fig. 1 and the mean changes in $\mathrm{K}_{\text {Mean }}$ and $\mathrm{K}_{\mathrm{Max}}$ are demonstrated in Fig. 2. The mean depth of the central demarcation line at 1 month postoperatively as measured on anterior segment OCT examination was $186 \pm 19 \mu \mathrm{m}$.

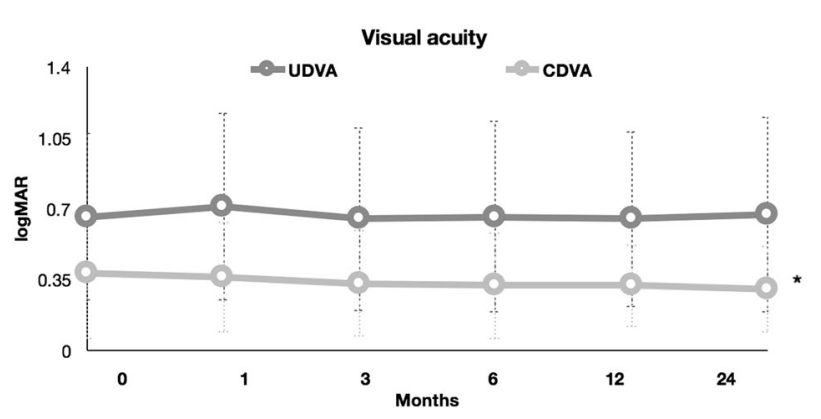

Fig. 1 The logMAR UDVA and CDVA after t-ACXL. There was no significant change in $\operatorname{logMAR}$ UDVA at any time point after treatment. The CDVA showed no significant change up to the 12 months time point, but showed significant improvement at 24 months compared to baseline $(0.38 \pm 0.32$ at baseline to $0.30 \pm 0.21$ at 24 months $* P \leq 0.01)$

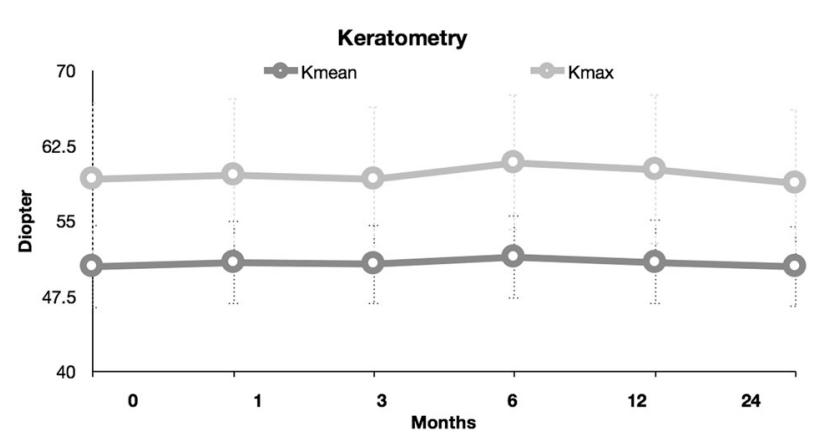

Fig. 2 The mean change in $\mathrm{K}_{\mathrm{Max}}$ and $\mathrm{K}_{\text {Mean }}$ following t-ACXL. There was no significant change in $\mathrm{K}_{\text {Max }}$ or $\mathrm{K}_{\text {Mean }}$ at any time point after t-ACXL

There were no significant differences between the year 1 and baseline and year 2 and baseline regarding mean UDVA, MRSE, $\mathrm{K}_{\text {Mean }}$, $\mathrm{K}_{\mathrm{Max}}$, anterior astigmatism, cornea thinnest point (CTP), asphericity, endothelial cell count and total corneal densitometry. Only one patient $(2.5 \%)$ showed evidence of progression at the end of the 2-year period, whilst six patients $(15 \%)$ exhibited a $\mathrm{K}_{\mathrm{Max}}$ reduction of $\geq 1.0$ D. Only one patient lost more than two lines of CDVA $(2.5 \%)$, with 10 patients $(25 \%)$ gaining two or more lines of CDVA at the final follow-up.

There was also no statistically significant difference in the corneal asymmetry indices; index of surface variance (ISV), index of vertical asymmetry (IVA), keratoconus index $(\mathrm{KI})$, centre keratoconus index (CKI), index of height asymmetry (IHA) and index of height decentration (IHD) following treatment. However, CDVA was significantly improved at the year 2 visit $(0.30 \pm 0.21$ $\log$ MAR) compared to the preoperative examination $(0.38$ \pm 0.32 logMAR) $(P<0.01)$.

The improvement in visual acuity was inversely correlated with preoperative CDVA $(r=-0.67, P<0.01)$. The improvement in visual acuity was also inversely correlated 
Table 2 Mean change in visual, refractive and tomographic parameters over 24 months

\begin{tabular}{llllll}
\hline & Preoperative & 12 months & 24 months & P1 $^{\mathrm{a}}$ & P2 $^{\mathrm{b}}$ \\
\hline UDVA (logMAR) & $0.81 \pm 0.40(0.12-1.58)$ & $0.81 \pm 0.40(0.18-1.60)$ & $0.88 \pm 0.41(0.06-2.08)$ & 0.97 & 0.08 \\
CDVA (logMAR) & $0.38 \pm 0.32(0.12-1.58)$ & $0.32 \pm 0.20(0.18-1.60)$ & $0.30 \pm 0.21(0.06-2.00)$ & 0.12 & $<\mathbf{0 . 0 1}$ \\
MRSE (D) & $-5.32 \pm 5.00(-19.25-1.00)$ & $-5.01 \pm 5.47(-16.25-1.87)$ & $-4.67 \pm 5.40(-16.37-1.25)$ & 0.54 & 0.19 \\
K $_{\text {Mean }}$ (D) & $50.45 \pm 3.99(44.3-61.5)$ & $50.82 \pm 4.05(44.3-61.3)$ & $50.36 \pm 3.87(44.2-61.6)$ & 0.87 & 0.93 \\
K Max (D) & $59.13 \pm 7.40(46.6-80.7)$ & $60.08 \pm 7.24(48.1-78.6)$ & $58.73 \pm 7.13(47.1-80.6)$ & 0.96 & 0.78 \\
Anterior astigmatism (D) & $4.27 \pm 2.44(0.4-9.0)$ & $4.48 \pm 2.24(0.7-7.9)$ & $4.31 \pm 2.19(1.7-9.8)$ & 0.74 & 0.98 \\
CTP (um) & $419 \pm 42(335-529)$ & $421 \pm 48(305-530)$ & $434 \pm 47(293-538)$ & 0.93 & 0.21 \\
Asphericity (Q) & $-1.02 \pm 0.51(-1.89$ to -0.22$)$ & $-1.03 \pm 0.53(-1.83$ to -0.14$)$ & $-1.00 \pm 0.52(-1.95$ to -0.26$)$ & 0.82 & 0.96 \\
ISV & $97.12 \pm 42.58(25-192)$ & $99.08 \pm 39.49(44-194)$ & $93.68 \pm 40.9(27-175)$ & 0.82 & 0.70 \\
IVA & $0.93 \pm 0.50(0.11-1.77)$ & $0.94 \pm 0.49(0.13-1.72)$ & $0.89 \pm 0.46(0.06-1.70)$ & 0.71 & 0.68 \\
KI & $1.27 \pm 0.16(0.99-1.71)$ & $1.28 \pm 0.17(0.87-1.75)$ & $1.25 \pm 0.15(0.92-1.58)$ & 0.89 & 0.61 \\
CKI & $1.08 \pm 0.06(0.98-1.22)$ & $1.08 \pm 0.07(0.94-1.23)$ & $1.07 \pm 0.06(0.94-1.23)$ & 0.38 & 0.68 \\
IHA & $31.9 \pm 25.24(1.0-101.3)$ & $39.38 \pm 25.86(0.1-93.3)$ & $42.33 \pm 36.1(0-164.2)$ & 0.12 & 0.20 \\
IHD & $0.14 \pm 0.08(0.00-0.34)$ & $0.15 \pm 0.08(0.04-0.33)$ & $0.13 \pm 0.08(0.00-0.33)$ & 0.79 & 0.82 \\
\hline
\end{tabular}

Significant $p$-values are given in bold

$C T P$ Cornea thinnest point, ISV index of surface variance, IVA index of vertical asymmetry, $K I$ keratoconus index, $C K I$ center keratoconus index, IHA index of height asymmetry, IHD index of height decentration

${ }^{\text {a }}$ Paired $t$-test, comparison of preoperative and year 1 values

${ }^{\mathrm{b}}$ Paired $t$-test, comparison of preoperative and year 2 values

with the preoperative $\mathrm{K}_{\mathrm{Max}}(r=-0.52$ and $P<0.01)$. Table 2.

\section{Discussion}

Keratoconus is a debilitating condition with a disproportionately high impact on public health resources and vision-specific quality of life [24]. CXL has revolutionised the management of keratoconus in recent years by effectively stabilising the underlying ectatic process and in some cases reversing the disease as quantified by key topographic, refractive and visual outcomes [7, 25]. Transepithelial crosslinking is a relatively recent modification to the conventional CXL procedure, which aims to offer numerous advantages by reducing postoperative discomfort, accelerating visual recovery and decreasing the risk of postoperative complications associated with epithelial debridement [26]. It should, however, be noted that the transepithelial procedure does not eliminate the risk of complications such as epithelial sloughing, oedema and diffuse punctate epitheliopathy [27].

Previous studies have demonstrated that in contrast to the conventional procedure, transepithelial crosslinking leads to no detectable increase in collagen fibre diameter [28] and a more limited apoptotic effect on stromal keratocytes [29] suggesting the transepithelial protocol has a more superficial effect on the cornea. There are conflicting reports in the literature regarding the efficacy of transepithelial $\mathrm{CXL}$ with some authors reporting stability of the ectatic process after treatment $[18,30]$, and others reporting progression of the disease entity in a significant proportion of patients [3133]. There are also conflicting reports regarding the efficacy of transepithelial CXL when compared to epithelium-off CXL with two randomised trials demonstrating superior efficacy of the epithelium-off procedure [34, 35] and one randomised trial reporting similar outcomes between the two treatment modalities [36]. This is unsurprising given the difference in patient populations, disease severity and the diversity of surgical protocols to ensure penetration of the riboflavin with a molecular weight $376 \mathrm{~g} / \mathrm{mol}$ through the lipophilic cornea and its epithelial tight junctions. These protocols include the use of molecular enhancers such as BAK, ethylenediaminetetraacetic acid or trometamol to loosen epithelial tight junctions and iontophoresis to improve the diffusion of the negatively charged riboflavin molecule through an intact epithelial layer [7].

The inherent technical difficulties associated with the transepithelial procedure led to some questioning the clinical efficacy of this protocol, but a recent meta-analysis has shown no significant difference between transepithelial and conventional crosslinking in visual acuity, flattest keratometry value, steepest keratometry value, spherical equivalent, or endothelial cell density [37]. The meta-analysis did, however, suggest that the transepithelial procedure has a protective influence on corneal thickness but leads to less postoperative corneal flattening compared to conventional crosslinking [37]. 
Pulsed, accelerated crosslinking is a more novel modification theoretically allowing for a higher stromal oxygen concentration and potential optimisation of the balance between the type II (aerobic) process which typically serves only as a transient and initiating role in the crosslinking process and the type I (anaerobic) photochemical reaction [15]. Pulsed CXL has shown promising preclinical results with porcine corneas crosslinked using the pulsed irradiation proving more resistant to enzymatic digestion than those treated with the continuous irradiation [38]. However, the exact duration of UV-A pulsing is yet to be elucidated as the typical rate of oxygen depletion in a type II photochemical reaction is 15-20 s [15] and physiological tissue oxygen replenishment is only achieved 3 min following discontinuation of UV-A irradiation [39]. This has led to some investigators questioning the efficacy of the current pulsed crosslinking protocols and advocating for an increase in the pulse cycle or a 30-40\% increase in UV-A dosage to optimise the crosslinking process [40, 41].

In this study, we analysed the effect of t-ACXL on patients with progressive keratoconus. Our results demonstrate that t-ACXL can effectively halt the progression of the ectatic process as measured by key tomographic indices and deliver a statistically significant improvement in CDVA, after a 24-month follow-up period. We hypothesise that the improvement in CDVA which was not accompanied by a change in MRSE or keratometric parameters, is a result of corneal epithelium remodelling, which leads to a decrease in surface irregularities [42]. We encountered no serious postoperative complications such as corneal haze, oedema, infection or endothelial loss throughout the followup period. Pain or foreign body sensation was commonly reported in the first 3 days following t-ACXL but resolved by ten days in all cases.

To our knowledge, there is only one other study reporting on the outcomes of t-ACXL. Tian et al. retrospectively analysed the outcomes of 17 paediatric patients undergoing t-ACXL and reported no statistically significant differences in $\mathrm{K}_{\mathrm{Max}}$, CTP, CDVA or MRSE after 12 months [43]. The results of this small, retrospective study were encouraging with the authors concluding that $\mathrm{t}-\mathrm{ACXL}$ is a safe treatment for keratoconus which partially prevents disease progression.

Two other studies have reported the outcomes of accelerated but non-pulsed CXL. Aixinjueluo et al. published on 30 eyes undergoing accelerated transepithelial CXL and reported a significant decrease in $\mathrm{K}_{\mathrm{Max}}$, mean keratometry, CTP and a significant improvement in CDVA after a follow-up period of 12 months [44]. Kir et al. published on 48 eyes undergoing accelerated transepithelial CXL and reported no significant changes in MRSE, CDVA and tomographic indices, but did show a significant increase in CTP after 24 months of follow-up [20].
Our results suggest that t-ACXL appears to halt the progression of ectasia in patients with thin corneas and leads to improved CDVA after a period of 2 years, but the procedure does not lead to a flattening effect of the cornea, nor does it increase the corneal thickness at the thinnest point. The demarcation line following CXL, has been proposed to be an indirect indicator of crosslinking efficacy and is in all probability, a clinical manifestation of the depth of CXL, a transition zone between the treated anterior corneal stroma and the untreated posterior layers, that occurs as a result of a change in the stromal refractive index, an increase in collagen fibre diameter, and fibrillar spacing [7]. The mean demarcation line following t-ACXL in this study was $186 \pm 19 \mu \mathrm{m}$ which is similar to other published reports following accelerated transepithelial CXL [44]. The demarcation line following t-ACXL however, appears to be more superficial than other protocols such as conventional CXL $(275 \mu \mathrm{m})$, accelerated-epithelium-off CXL $(279 \mu \mathrm{m})$ and transepithelial iontophoresis assisted ACXL $(235 \mu \mathrm{m})$ [45].

Although prospective in design, our study has a number of potential limitations including a modest sample size, lack of a control group and in vivo morphological evaluation. A large, well-designed randomised controlled trial comparing t-ACXL with other CXL protocols is required to establish its non-inferiority and confirm its superiority in complication profile.

In conclusion, our results suggest that t-ACXL appears to be an effective method of halting the progression of keratoconus over a 24-month follow-up period. This method has the advantages of both accelerated and transepithelial protocols including reduced surgical times, increased patient comfort and a reduced postoperative complication profile. We would, however, advise caution with regards to using this crosslinking protocol routinely and we currently employ this method of CXL in patients with thinner corneas, those who may have difficulty in cooperating with the epithelium-off CXL protocol, and patients who may not adhere to postoperative treatment and follow-up.

\section{Summary}

\section{What was known before}

- Clinical studies have reported short-term favourable outcomes with both accelerated and transepithelial crosslinking protocols.

\section{What this study adds}

- Transepithelial, pulsed, accelerated corneal collagen crosslinking is a safe and effective method for halting 
the progression of keratoconus over a 24-month followup period and leads to an improvement in corrected distance visual acuity.

Acknowledgements The authors report no conflicts of interest. We warrant that this paper is original and has not been in part or in whole published in another journal.

\section{Compliance with ethical standards}

Conflict of interest The authors declare that they have no conflict of interest.

Publisher's note: Springer Nature remains neutral with regard to jurisdictional claims in published maps and institutional affiliations.

\section{References}

1. Krachmer JH, Feder RS, Belin MW. Keratoconus and related noninflammatory corneal thinning disorders. Surv Ophthalmol. 1984;28:293-322.

2. Davidson AE, Hayes S, Hardcastle AJ, Tuft SJ. The pathogenesis of keratoconus. Eye. 2014;28:189-95.

3. Wollensak G, Spoerl E, Seiler T. Riboflavin/ultraviolet-a-induced collagen crosslinking for the treatment of keratoconus. Am J Ophthalmol. 2003;135:620-7.

4. Spoerl E, Huhle M, Seiler T. Induction of cross-links in corneal tissue. Exp Eye Res. 1998;66:97-103.

5. McCall AS, Kraft S, Edelhauser HF, Kidder GW, Lundquist RR, Bradshaw HE, et al. Mechanisms of corneal tissue cross-linking in response to treatment with topical riboflavin and long-wavelength ultraviolet radiation (UVA). Investig Ophthalmol Vis Sci. 2010;51:129-38.

6. Wollensak G, Spoerl E, Seiler T. Stress-strain measurements of human and porcine corneas after riboflavin-ultraviolet-A-induced cross-linking. J Cataract Refract Surg. 2003;29:1780-5.

7. Ziaei M, Barsam A, Shamie N, Vroman D, Kim T, Donnenfeld $\mathrm{ED}$, et al. Reshaping procedures for the surgical management of corneal ectasia. J Cataract Refract Surg. 2015;41:842-72.

8. Medeiros CS, Giacomin NT, Bueno RL, Ghanem RC, Moraes HV Jr., Santhiago MR. Accelerated corneal collagen crosslinking: technique, efficacy, safety, and applications. J Cataract Refract Surg. 2016;42:1826-35.

9. Wen D, Li Q, Song B, Tu R, Wang Q, O’Brart DPS, et al. Comparison of standard versus accelerated corneal collagen crosslinking for keratoconus: a meta-analysis. Invest Ophthalmol Vis Sci. 2018;59:3920-31.

10. Hammer A, Richoz O, Arba Mosquera S, Tabibian D, Hoogewoud F, Hafezi F. Corneal biomechanical properties at different corneal cross-linking (CXL) irradiances. Invest Ophthalmol Vis Sci. 2014;55:2881-4

11. Mazzotta C, Traversi C, Caragiuli S, Rechichi M. Pulsed vs continuous light accelerated corneal collagen crosslinking: in vivo qualitative investigation by confocal microscopy and corneal OCT. Eye. 2014;28:1179-83.

12. Shajari M, Kolb CM, Agha B, Steinwender G, Muller M, Herrmann E, et al. Comparison of standard and accelerated corneal cross-linking for the treatment of keratoconus: a meta-analysis. Acta Ophthalmol. 2019;97:e22-35.

13. Liu Y, Liu Y, Zhang YN, Li AP, Zhang J, Liang QF, et al. Systematic review and meta-analysis comparing modified cross- linking and standard cross-linking for progressive keratoconus. Int J Ophthalmol. 2017;10:1419-29.

14. Turkcu UO, Yuksel N, Novruzlu S, Yalinbas D, Bilgihan A, Bilgihan K. Protein oxidation levels after different corneal collagen cross-linking methods. Cornea. 2016;35:388-91.

15. Kamaev P, Friedman MD, Sherr E, Muller D. Photochemical kinetics of corneal cross-linking with riboflavin. Invest Ophthalmol Vis Sci. 2012;53:2360-7.

16. Lesniak SP, Hersh PS. Transepithelial corneal collagen crosslinking for keratoconus: six-month results. J Cataract Refract Surg. 2014;40:1971-9.

17. De Bernardo M, Capasso L, Tortori A, Lanza M, Caliendo L, Rosa N. Trans epithelial corneal collagen crosslinking for progressive keratoconus: 6 months follow up. Cont Lens Anterior Eye. 2014;37:438-41.

18. Filippello M, Stagni E, O’Brart D. Transepithelial corneal collagen crosslinking: bilateral study. J Cataract Refract Surg. 2012;38:283-91.

19. Salman AG. Transepithelial corneal collagen crosslinking for progressive keratoconus in a pediatric age group. J Cataract Refract Surg. 2013;39:1164-70.

20. Kir MB, Turkyilmaz K, Oner V. Transepithelial high-intensity cross-linking for the treatment of progressive keratoconus: 2-year outcomes. Curr Eye Res. 2017;42:28-31.

21. Ziaei, M., et al., Prospective two-year study of clinical outcomes following epithelium-off pulsed versus continuous accelerated corneal crosslinking for keratoconus. Clin Exp Ophthalmol. 2019.

22. Hashemi H, Yekta A, Khabazkhoob M. Effect of keratoconus grades on repeatability of keratometry readings: comparison of 5 devices. J Cataract Refract Surg. 2015;41:1065-72.

23. de Luis Eguileor B, Escudero Argaluza J, Pijoan Zubizarreta JI, Santamaria Carro A, Etxebarria Ecenarro J. Evaluation of the reliability and repeatability of scheimpflug system measurement in keratoconus. Cornea. 2018:37:177-81.

24. Kymes SM, Walline JJ, Zadnik K, Gordon MO, Collaborative Longitudinal Evaluation of Keratoconus study g. Quality of life in keratoconus. Am J Ophthalmol. 2004;138:527-35.

25. Spoerl E, Wollensak G, Seiler T. Increased resistance of crosslinked cornea against enzymatic digestion. Curr Eye Res. 2004;29:35-40.

26. Caporossi A, Mazzotta C, Baiocchi S, Caporossi T, Paradiso AL. Transepithelial corneal collagen crosslinking for keratoconus: qualitative investigation by in vivo HRT II confocal analysis. Eur J Ophthalmol. 2012;22 (Suppl 7):S81-8.

27. Chow SSW, Chan TCY, Wong IYH, Fan MCY, Lai JSM, Ng ALK. Early epithelial complications of accelerated trans-epithelial corneal crosslinking in treatment of keratoconus: a case series. Int Ophthalmol. 2018;38:2635-8.

28. Mencucci R, Paladini I, Sarchielli E, Favuzza E, Vannelli GB, Marini M. Transepithelial riboflavin/ultraviolet. a corneal crosslinking in keratoconus: morphologic studies on human corneas. Am J Ophthalmol. 2013;156:874-884 e871.

29. Mazzotta C, Caporossi T, Denaro R, Bovone C, Sparano C, Paradiso A, et al. Morphological and functional correlations in riboflavin UV A corneal collagen cross-linking for keratoconus. Acta Ophthalmol. 2012;90:259-65.

30. Leccisotti A, Islam T. Transepithelial corneal collagen crosslinking in keratoconus. J Refract Surg. 2010;26:942-8.

31. Buzzonetti L, Petrocelli G. Transepithelial corneal cross-linking in pediatric patients: early results. J Refract Surg. 2012;28:763-7.

32. Caporossi A, Mazzotta C, Paradiso AL, Baiocchi S, Marigliani D, Caporossi T. Transepithelial corneal collagen crosslinking for progressive keratoconus: 24-month clinical results. J Cataract Refract Surg. 2013;39:1157-63.

33. Soeters N, Wisse RP, Godefrooij DA, Imhof SM, Tahzib NG. Transepithelial versus epithelium-off corneal cross-linking for the 
treatment of progressive keratoconus: a randomized controlled trial. Am J Ophthalmol. 2015;159:821-8 e823.

34. Cerman E, Toker E, Ozarslan Ozcan D. Transepithelial versus epithelium-off crosslinking in adults with progressive keratoconus. J Cataract Refract Surg. 2015;41:1416-25.

35. Rush SW, Rush RB. Epithelium-off versus transepithelial corneal collagen crosslinking for progressive corneal ectasia: a randomised and controlled trial. Br J Ophthalmol. 2017;101:503-8.

36. Nawaz S, Gupta S, Gogia V, Sasikala NK, Panda A. Trans-epithelial versus conventional corneal collagen crosslinking: a randomized trial in keratoconus. Oman J Ophthalmol. 2015;8:9-13.

37. Zhang X, Zhao J, Li M, Tian M, Shen Y, Zhou X. Conventional and transepithelial corneal cross-linking for patients with keratoconus. PLoS ONE. 2018;13:e0195105.

38. Aldahlawi NH, Hayes S, O'Brart DP, Akhbanbetova A, Littlechild SL, Meek KM. Enzymatic resistance of corneas crosslinked using riboflavin in conjunction with low energy, high energy, and pulsed UVA irradiation modes. Invest Ophthalmol Vis Sci. 2016;57:1547-52.

39. Sachdev GS, Sachdev M. Recent advances in corneal collagen cross-linking. Indian J Ophthalmol. 2017;65:787-96.

40. Kymionis GD, Tsoulnaras KI, Grentzelos MA, Liakopoulos DA, Tsakalis NG, Blazaki SV, et al. Evaluation of corneal stromal demarcation line depth following standard and a modifiedaccelerated collagen cross-linking protocol. Am J Ophthalmol. 2014;158:671-5 e671.

41. Sherif AM. Accelerated versus conventional corneal collagen cross-linking in the treatment of mild keratoconus: a comparative study. Clin Ophthalmol. 2014;8:1435-40.

42. Ziaei M, Meyer J, Gokul A, Vellara H, McGhee CNJ. Direct measurement of anterior corneal curvature changes attributable to epithelial removal in keratoconus. J Cataract Refract Surg. 2018;44:71-7.

43. Tian M, Jian W, Sun L, Shen Y, Zhang X, Zhou X. One-year follow-up of accelerated transepithelial corneal collagen crosslinking for progressive pediatric keratoconus. BMC Ophthalmol. 2018;18:75.

44. Aixinjueluo W, Usui T, Miyai T, Toyono T, Sakisaka T, Yamagami S. Accelerated transepithelial corneal cross-linking for progressive keratoconus: a prospective study of 12 months. $\mathrm{Br}$ J Ophthalmol. 2017;101:1244-9.

45. Spadea L, Di Genova L, Tonti E. Corneal stromal demarcation line after 4 protocols of corneal crosslinking in keratoconus determined with anterior segment optical coherence tomography. J Cataract Refract Surg. 2018;44:596-602. 